\title{
Digital Music Libraries - Research and Development
}

\author{
David Bainbridge \\ Dept. of Computer Science \\ University of Waikato \\ Hamilton, New Zealand \\ $+64-7-838-4407$ \\ D.Bainbridge@cs.waikato.ac.nz
}

\author{
Andrew P. Dillon \\ School of Library and Information \\ Science, Indiana University \\ Bloomington, IN, 47405, USA \\ +1-812-855-3249 \\ adillon@indiana.edu
}

\author{
Michael Fingerhut \\ Director, Multimedia Library \\ Ircam, Centre Pompidou \\ Paris, France \\ +33144784853 \\ mf@ircam.fr
}

\author{
Gerry Bernbom \\ Research \& Academic Computing \\ Indiana University \\ Bloomington, IN, 47405, USA \\ +1-812-856-5595 \\ bernbom@ indiana.edu \\ (Moderator) \\ Matthew Dovey \\ Libraries Automation Service \\ University of Oxford \\ Oxford, OX1, 3LU, UK \\ +44-1865-278272 \\ matthew.dovey@las.ox.ac.uk
Ichiro Fujinaga
Peabody Conservatory of Music
Johns Hopkins University
Baltimore, MD, 21202 USA
+1-410-659-8107
ich@peabody.jhu.edu

\author{
Mary Wallace Davidson \\ William and Gayle Cook Music Library \\ Indiana University \\ Bloomington, IN, 47405, USA \\ +1-812-855-2970 \\ mdavidso@indiana.edu
}

\begin{abstract}
Digital music libraries provide enhanced access and functionality that facilitates scholarly research and education. This panel will present a report on the progress of several major research and development projects in digital music libraries.
\end{abstract}

\section{Categories and Subject Descriptors}

J.5 [Arts and Humanities]-performing arts; H.3.7 [Information Storage and Retrieval]: Digital librariescollection, dissemination, systems issues, user issues.

\section{Keywords}

Digital music libraries, digital libraries, music libraries, music information retrieval, optical music recognition.

\section{INTRODUCTION}

Projects represented and the some of the issues they will address are as follows.

The Indiana University Digital Music Library project $<$ http://dml.indiana.edu/ $>$ :

- Integrating digital music library collections with interactive music instructional applications, and addressing issues of metadata and intellectual property that are unique to music.

- Designing a system architecture to support content management, multimedia delivery, and interactive instruction.

Copyright is held by the author/owner(s).

$J C D L$ '00, June 24-28, 2001, Roanoke, Virginia, USA.

ACM 1-58113-345-6/01/0006.
- Including users in the design process and applying human computer interaction methods to the systems development methodology.

The Lester S. Levy Collection of Sheet Music Project at The Johns Hopkins University <http://levysheetmusic.mse.jhu.edu/>:

- Developing optical music recognition software to create audio files and full-text lyrics from scanned sheet music.

- Developing workflow management tools to reduce and focus human labor in the digitization process.

The Ircam Multimedia Library $<$ http://mediatheque.ircam.fr $>$ in Paris, which combines a lending library with an archive of concert recordings and a digital library.

Music Digital Libraries and Library Automation at University of Oxford, including the OMRAS (Online Music Retrieval and Searching) project $<$ http://www.omras.org/>:

- Combining content-based music retrieval with online library catalog searching.

- Developing efficient content-based searching and retrieval of musical information from online databases stored in a variety of formats ranging from encoded scores to digital audio

The MELDEX digital music library in the New Zealand Digital Library (NZDL) project <http://www.nzdl.org/>:

- Developing software for managing digital music content including optical music recognition and content-based retrieval. 
NOTE: The IU Digital Music Library Project and the Lester S. Levy Sheet Music Project receive funding from the NSF Digital Libraries Initiative-Phase Two (DLI2). The OMRAS project receives funding from the NSF/JISC International Digital Libraries Initiative.

\section{PANELISTS' STATEMENTS 2.1 David Bainbridge}

At the New Zealand Digital Library project we are interested in how software for a digital music library is similar to that used in other forms of digital library. The main areas of study are document acquisition, searching, browsing, cross-referencing, and presentation -- in broad terms, the life-cycle of a digital library. We are also interested in usability issues and "summarization" of music. Meldex (http://www.nzdl.org/musiclib), a working digital music library system, is where we demonstrate techniques that culminate from our research, and it includes examples from each of the life-cycle categories above.

\subsection{Mary Wallace Davidson}

"Creating a Digital Music Library," the title of the IU's DML project as proposed, forces us to rethink traditional concepts about music libraries. Our first task is to define what we mean by creating a digital music library, as opposed to providing access to a music collection. "Creating" implies collecting, but collecting what, for whom? What will our users do, or want to do with the digital possibilities afforded? What added value can we (afford to) provide? The answers are based on inherent or rationalized musical relationships among or within these materials that we want to describe and express in searchable schemes (metadata). As always, we want our digital music library to incorporate musical objects whether or not they are currently protected by copyright, and to preserve them for future use.

\subsection{Andrew P. Dillon}

The user experience of digital information has often proved problematic, with navigation difficulties, lower comprehension, slower reading and greater cognitive effort being reported. In the IU DML project we are attempting to address these concerns upfront with continuous user testing and involvement in the design process. I will outline how user centered design is playing out in the design process and describe the obstacles faced in gaining user input in a form that can help shape emerging designs.

\subsection{Matthew Dovey}

Oxford University Libraries and the Music Faculty at Oxford are currently embarking on a number of music digital library initiatives including digitization of the University Libraries' assets and work on digital audio delivery. One of the more technically interesting areas is the objective of integrating these initiatives with our current library system. We are combining research into music information retrieval conducted by Kings College London and the University of Massachusetts at Amherst as part of project OMRAS (Online Music Retrieval and Searching) with work at Oxford in java-based Z39.50 tools (JAFER: http://www.lib.ox.ac.uk/jafer) to integrate these initiatives into our main library catalogue. This work will lead to the ability to search the Oxford Libraries catalogue by musical content queries as well as text queries and also will allow retrieval of the electronic forms of the material (both images and audio). We are working in collaboration with the Johns Hopkins University to provide the ability to extract musical data from images via Optical Music Recognition and with Kings College London to extract musical data from audio via Digital Signal Processing. We are also working with various partners such as the Indiana Digital Music Library project, Naxos, Computer Associates and the Oxford Technology-Assisted Lifelong Learning project (http://www.tall.ox.ac.uk) to establish ways of integrating this material into the learning environment.

\subsection{Jon W. Dunn}

The goals of the IU Digital Music Library project raise a number of technical system architecture and design challenges. Principal among them is the development of a data model and metadata scheme to represent the complex relationships between the various representations (sound, score, MIDI, video, etc.) of a given musical work in order to enable needed search, retrieval, and navigation capabilities. Equally important is the creation of an extensible software architecture for the system, to enable creation of user interface applications which use library content and services to meet the needs of a variety of user groups, including students, teachers, and researchers. In addition, other challenges include dealing with problems of authentication/access control, rights management, and network delivery over connections which vary in quality.

\subsection{Michael Fingerhut}

The Ircam Multimedia Library combines a lending library with an archive of concert recordings and a digital library. It uses a traditional library system for most of the material, supplemented by various extensions to allow for the integration of the online material. The online availability of material (recordings, scores, film, text) allows for the creation of "higher-level" pedagogical documents (musical analyses, score study tools, and others). We are currently studying evolutions of the underlying system towards one tightly integrating various types of metadata (*MARC, Dublin Core, MPEG-7, etc.), so as to preserve and take advantage of the mechanisms of library systems (e.g., authority control) as well as allow for more powerful search and access tools.

\subsection{Ichiro Fujinaga}

The Lester S. Levy Collection of Sheet Music at Johns Hopkins University represents one of the largest collections of sheet music available online, comprised of nearly 30,000 pieces of music and associated cover art. One of the missions of the Levy project is to reduce costs for large collection ingestion by creating a suite of open-source processes, tools, and interfaces for workflow management. A major component of the system is the optical music recognition (OMR) software, which uses a combination of heuristic and exemplar-based classifiers. Compared to commercial systems, our OMR software is: open-source, platform independent, able to "learn" different types of musical and other symbols, able to run in batch mode, able to extract lyrics, and able to produce a searchable logical representation of the score for sound generation, musical searching, and musicological research. Other components include the incorporation of an XML structure for metadata and the enhanced searching capabilities through development and application of a fast, disk-based search engine for lyrics and music. 


\subsection{Eric J. Isaacson}

Having access to various representations of music (audio, scanned scores, encoded scores, etc.) will be invaluable to the classroom music faculty. But to make full use of those resources, instructors have to be able to access and coordinate those materials in a dynamic fashion. We therefore need to provide authoring tools that will enable instructors to create classroom presentations and student activities that can make use of these materials in a pedagogically effective manner. Such tools will enable the instructor to do such things as play and display segments of stored examples, possibly from multiple recordings or printed editions, annotate them using any number of conventional systems, and create representations such as timelines or analytic "reductions." We will describe these needs, and the authoring and user environment designed to meet them in Indiana University's Multimedia Music Theory Teaching Project. 